\title{
Mortalidade por câncer em trabalhadores de companhia geradora de eletricidade do Estado de São Paulo, Brasil*
}

\section{Cancer mortality among electricity utility workers in a Southeastern region Brazil}

\section{Inês E. Mattos e Sérgio Koifman}

Departamento de Epidemiologia e Métodos Quantitativos em Saúde da Escola Nacional de Saúde Pública. Fundação Oswaldo Cruz. Rio de Janeiro, RJ - Brasil

\begin{abstract}
Resumo
Realizou-se a análise exploratória de um conjunto de atestados de óbito de eletricitários do Estado de São Paulo (Brasil), através da Razão de Mortalidade Proporcional (PMR) e da Razão de Mortalidade Proporcional por Câncer (PCMR). Foi observado um discreto excesso de mortalidade por todas as localizações de câncer (PMR 1,11; IC 95\% 0,91-1,35), estatisticamente não significativo. Encontrou-se elevação da PCMR, estatisticamente significativa, para câncer de laringe (PCMR 2,04; IC 95\% 1,05-4,20). Um excesso de óbitos, embora sem significação estatística, foi observado para cavidade oral/faringe, próstata, bexiga, cérebro e doença de Hodgkin. A análise por categorias de exposição estimada a campos magnéticos mostrou PCMRs persistentemente elevadas para cancêr de bexiga (PCMR 4,17; IC 95\% 1,35-9,72), cérebro (PCMR 3,77; IC 95\% 1,02-9,65) e doença de Hodgkin (PCMR 5,55; IC 95\% 1,14-16,21), no grupo com exposição provavelmente alta. Uma comparação com a mortalidade por câncer, de um grupo de trabalhadores petroquímicos, mostrou mortalidade elevada por neoplasma de laringe (PCMR 3,51; IC 95\% 1,75-6,28) e bexiga (PCMR 7,53; IC 95\% 3,02-15,51). Para tumores de cérebro, observou-se PCMR de 0,74 (IC 95\% 0,27-1,61).
\end{abstract}

Exposição ocupacional, efeitos adversos. Neoplasias, mortalidade. Eletricidade, efeitos adversos.

\begin{abstract}
s
A number of epidemiologic studies have observed an association between exposure to 50-60 Hz electromagnetic fields and the development of specific types of cancer. In Brazil, a preliminary report from a study of electricity facility workers in Rio de Janeiro (RJ) has mentioned relatively similar results. An exploratory analysis of death certificates obtained from a sample of
\end{abstract}

\footnotetext{
* Extraído da dissertação de mestrado "Mortalidade por câncer em trabalhadores de companhia geradora de eletrioidade em São Paulo", apresentada à Escola Nacional de Saúde Pública/FIOCRUZ, 1993, por I.E. Mattos.

Correspondência para/Correspondence to: Inês E. Mattos - Departamento de Epidemiologia e Métodos Quantitantivos em Saúde da Escola Nacional de Saúde Pública/FIOCRUZ. Rua Leopoldo Bulhões, 1480 - $8^{\circ}$ andar - Manguinhos - 21041-210 Rio de Janeiro, RJ - Brasil. E-mail: Ines@ax.apc.org Recebido em 19.1.1996. Aprovado em 3.6.1996.
} 
electricity workers in S. Paulo was made. Data was analysed by using the Proportional Mortality Ratio (PMR) and the Proportional Cancer Mortality Ratio (PCMR). A slightly elevated all-sites cancer mortality was observed among these workers (PMR 1.11; 95\% CI 0.91-1.35). Site specific analysis has shown a statistically significant higher mortality of laryngeal cancer (PCMR 2.04; 95\% CI 1.05-4.20). An excess of deaths was also seen for cancers of the buccal cavity/pharynx, prostate, bladder, brain and Hodgkin's disease, although the results lacked statistical significance. When analysed by categories of estimated exposure to magnetic fields, an excess of deaths from bladder cancer (PCMR 4.17; 95\% CI 1.35-9.72), neoplasms of the brain (PCMR 7.7; 95\% CI 1.02-9.65) and Hodgkin's disease (PCMR 5.55; 95\% CI 1.14-16.21) was observed in the group with probably higher exposure to EMF. A comparison of cancer mortality between these workers and petrochemical employees has shown a higher PCMR for larynx tumours (PCMR 3.51; 95\% CI 3.02-15.51) and bladder cancer (PCMR 7.53; 95\% CI 3.02-15.51). For brain tumours, however, a PCMR of 0,74 (95\% CI 0.27-1.61) was noted. Although restrictions related to sample size in the study and the lack of information about known confounders must be considered, the results of this study do not fully disagree with others previously mentioned in the literature.

Occupational exposure, adverse effects. Neoplasms, mortality. Electricity, adverse effects.

\section{INTRODUÇÃO}

A possibilidade de uma associação causal entre campos eletromagnéticos de baixa freqüência (CEM) e câncer foi apresentada pela primeira vez em 1979, em um estudo epidemiológico de tipo caso-controle sobre neoplasias na infância ${ }^{80}$, realizado em Denver, Estados Unidos. Um pouco mais tarde, essa questão também seria estudada por Milhan ${ }^{50}$ com relação à exposição ocupacional. A partir desses trabalhos, um número crescente de estudos epidemiológicos e experimentais têm examinado as possíveis relações entre câncer e CEM em populações humanas, ani-

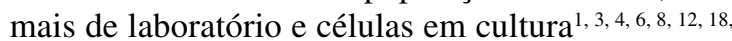
$24,58,61,62,63,64,65,68,74,76,81,85$

Embora não seja ainda possível um completo entendimento do processo de desenvolvimento de câncer em seres humanos, os modelos atualmente propostos para explicar a gênese de tumores consideram que existem múltiplos caminhos para a malignização ${ }^{29,78}$. Além dos mecanismos moleculares diretamente envolvidos na carcinogênese, existem diversos outros fatores que, embora não sejam cancerígenos, podem também atuar no processo e modificá-lo, sendo denominados de co-carcinógenos ${ }^{5,78}$.

Os estudos experimentais sobre os mecanismos envolvidos no aparecimento do câncer, a partir da exposição aos CEM, ainda se encontram numa fase inicial. As evidências biológicas disponíveis parecem indicar que sua ação se daria numa etapa mais tardia da carcinogênese, através de um efeito promotor ou co-promotor da doença ${ }^{28,29,38,42,69,78}$.

Uma das grandes dificuldades que envolve o trabalho experimental com CEM, bem como o desenvolvimento de estudos epidemiológicos mais precisos sobre seus efeitos na saúde da população, reside no fato de que a determinação dos parâmetros de

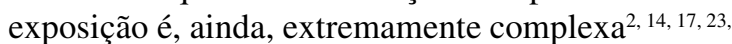
30, 35, 36, 46, 57. Essas dificuldades, que persistem ainda hoje, apesar dos avanços obtidos na área de medições de campos elétricos e magnéticos, levaram à utilização de medidas indiretas em muitos estudos epidemiológicos. A medida indireta mais utilizada, nos estudos ocupacionais, tem sido a ocupação, considerada em termos de atividades que envolvem exposição à eletricidade ${ }^{3,26,34,48,54,56,75,76,77,82,85}$.

Estudos de exposição ocupacional com medições diretas de $\mathrm{CEM}^{15,40}$ têm comprovado que algumas categorias de trabalhadores de companhias de eletricidade, como operadores de usinas ou subestações e eletricistas de diversas especialidades, estão, efetivamente, expostos a maiores intensidades de CEM. Embora sejam medidos os campos elétricos e magnéticos, acredita-se que os efeitos biológicos analisados estejam relacionados aos campos magnéticos, não só pelo fato de que eles praticamente não são atenuados por diversos tipos de estruturas mas, principalmente, porque é com esse tipo de radiação que os estudos epidemiológicos têm encontrado associações positivas ${ }^{21,45,63}$. 
No Brasil, resultados preliminares de um estudo epidemiológico de eletricitários do Município do Rio de Janeiro mostraram-se relativamente semelhantes aos encontrados em outros países ${ }^{39}$.

Considerando as diferentes características da população de cada país e as diversas formas em que a eletricidade é produzida e distribuída, julgou-se oportuno realizar uma análise exploratória da mortalidade por câncer em um grupo de eletricitários do Estado de São Paulo, SP.

\section{MATERIAL E MÉTODO}

O presente estudo é subproduto do projeto de pesquisa "Esperança de Vida e Mortalidade em Eletricitários do Rio de Janeiro", desenvolvido no período 1986/1992. Por ocasião desse projeto, foi identificado um grande número de atestados de óbito de trabalhadores de uma companhia elétrica. Um subgrupo de atestados de óbito de antigos trabalhadores do Estado de São Paulo constituiu-se no objeto de análise do presente trabalho.

Como período de estudo, foram estabelecidos os anos de 1975 a 1985, período em que havia disponibilidade de dados de mortalidade por câncer para a população masculina do Município de São Paulo, usada como comparação.

O subgrupo original de trabalhadores do Estado de São Paulo consistia de 719 atestados de óbito. Desse grupo, foram retirados 5 óbitos que haviam ocorrido fora do período do estudo. Posteriormente, uma revisão em antigos arquivos de pessoal da companhia de eletricidade levou à eliminação de mais 19 óbitos, por serem de indivíduos que não haviam trabalhado na mesma.

O grupo de estudo ficou, então, constituído por 695 atestados de óbito de trabalhadores eletricitários do Estado de São Paulo, do sexo masculino, falecidos no período de 1975 a 1985, com 20 ou mais anos de idade.

Cada um dos atestados de óbito foi codificado, em nível de três dígitos, de acordo com a causa básica de morte, utilizando a nona revisão da Classificação Internacional de Doenças (CID), sendo, posteriormente, agrupados segundo a Lista de Categorias de Três Algarismos, compondo os diversos grupos de causas de morte.

A Razão de Mortalidade Proporcional (PMR), ajustada por idade, foi calculada segundo a metodologia descrita por Monson ${ }^{52}$, sendo utilizados intervalos de confiança de $95 \%{ }^{43}$. Como população de referência, foi utilizada a população masculina, de 20 anos ou mais, do Município de São Paulo, já que a maioria dos indivíduos que compunha o universo de estudo (473, 68\% deste universo), havia residido no referido município. Como fonte dos dados de mortalidade, foram consultadas as publicações do Ministério da Saúde - Estatísticas de Mortalidade ${ }^{51}$, buscandose adequar os grupamentos etários utilizados para o cálculo da PMR àqueles empregados pelo Ministério da Saúde, para efeito de comparabilidade. Devido ao longo período de abrangência do estudo, no decorrer do qual ocorreram modificações na mortalidade pelos diversos grupos de causas, preferiu-se calcular as proporções de óbitos em duas etapas, obtendo-se a proporção mediana em cada uma delas. O objetivo dessa separação era o de tentar adequar as comparações efetuadas entre a mortalidade dos eletricitários com aquela observada na população masculina de São Paulo, levando em conta as mudanças ocorridas nesta última, ao longo do período de tempo analisado. A primeira etapa compreendeu os anos de 1975 a 1979 e, a segunda, de 1980 a 1985.

Em seqüência, foi calculada a "Mortality Odds Ratio" (MOR) medida de associação proposta por Miettinen e Wang ${ }^{49}$. Para essa análise, os atestados de óbito da amostra foram separados em apenas dois grupos, sendo o primeiro constituído por todos os óbitos de câncer (CID 140239) e, o segundo, pelas demais causas de morte. Para cada um desses grupos foi obedecida a mesma divisão etária anteriormente mencionada e utilizada a mesma população de referência. Para efeito do cálculo do MOR, o grupo de exposição foi formado pelo conjunto de óbitos ocorridos entre os eletricitários, sendo utilizados como controle todos os óbitos da população masculina do Município de São Paulo de 20 ou mais anos de idade, ocorridos no período de 1977 a 1985. Devido ao pequeno número de óbitos em cada faixa etária, não foi viável a realização da análise do MOR por tipos específicos de neoplasias.

A seguir, todos os atestados de óbito que mencionavam alguma neoplasia (CID 140-239) como causa básica de morte, em um total de 130, foram separados de acordo com as diferentes localizações anatômicas do câncer e segundo grupos de idade. Para essa fase, como estavam disponíveis dados de mortalidade por neoplasias da Fundação SEADE, optou-se por uma divisão em grupos etários decenais, não estratificando o grupo de 80 ou mais anos de idade.

A Razão de Mortalidade Proporcional por Câncer (CPMR), ajustada por idade, foi calculada para cada uma das localizações anatômicas, de acordo com a metodologia descrita por Monson ${ }^{52}$, sendo estabelecido, para cada resultado, o intervalo de confiança a $95 \%{ }^{43}$. Optou-se por manter a população masculina do Município de São Paulo como referência, pois a maior parte dos indivíduos falecidos com câncer $(62 \%)$ havia também residido naquele município. Por outro lado, uma comparação da mortalidade proporcional, para cada ano do período de estudo, mostrou que o percentual de óbitos por neoplasias na população do sexo masculino, de 20 ou mais anos de idade, é praticamente semelhante no município e no Estado de São Paulo, dados confirmados também por outros estudos ${ }^{25,33}$.

Os dados de mortalidade por câncer referentes aos anos de 1975 a 1985 foram obtidos na Fundação Sistema Estadual de Análise de Dados (SEADE). No cálculo das proporções de óbitos por cada tipo específico de câncer, devido aos mesmos motivos já mencionados, dividiu-se o período de estudo em duas fases: 1975 a 1979 e 1980 a 1985, sendo obtida a proporção da mortalidade mediana para cada período.

Procurou-se estabelecer a ocupação de cada um dos eletricitários falecidos com câncer, buscando identificar, de forma um pouco mais precisa, os indivíduos da amostra que desenvolviam atividades provavelmente relacio- 
nadas a uma maior exposição à eletricidade. Para essa finalidade, foram utilizados dados registrados no atestado de óbito e informações constantes da ficha individual de cada trabalhador da companhia. Devido a razões operacionais, não foi possível refazer toda a trajetória ocupacional dos indivíduos, sendo obtida, para o conjunto dos trabalhadores, apenas a ocupação que exerciam no momento da morte ou aposentadoria.

Estudos ocupacionais recentes realizaram medições de campos elétricos e magnéticos, detectando níveis diferentes de exposição, para determinados grupos de trabalhado$\mathrm{res}^{40}$. Os indivíduos da amostra foram classificados em três diferentes grupos de provável exposição aos campos magnéticos, com base nesses estudos e na opinião de especialistas brasileiros consultados, ligados à área de eletricidade (Tabela 1). A classificação nas categorias de exposição aos CEM foi feita sem o conhecimento prévio do tipo específico de câncer que causou a morte do indivíduo.

$\mathrm{Na}$ última etapa da análise foi realizada uma comparação entre a mortalidade por neoplasias dos eletricitários de São Paulo e aquela experimentada por funcionários de uma empresa do setor petroquímico. Os eletricitários e os trabalhadores petroquímicos formam dois grupos ocupacionais distintos, sujeitos a diferentes fatores de risco. Por outro lado, ambos se diferenciam da população em geral, pelo fato de terem passado, entre outros fatores, por processos de seleção para o trabalho, provavelmente apre- sentando, portanto, melhores condições de saúde que a população geral ("health worker effect") 9, 52, 53. Para essa comparação foram utilizados dados de mortalidade por câncer de um estudo epidemiológico de trabalhadores do setor petroquímico, cujo período de referência (1970 a $1985)^{47}$ abrange os anos do presente trabalho. Para que fosse possível a utilização desses dados, os grupos etários originais foram refeitos, com a exclusão dos óbitos ocorridos a partir de 80 anos, que não foram analisados no estudo mencionado, sendo calculadas as CPMRs, ajustadas por idade, para cada tipo específico de neoplasia, tendo como referência as proporções encontradas entre os trabalhadores petroquímicos.

\section{RESULTADOS}

A Tabela 2 mostra a PMR por causa, ajustada por idade. Verifica-se uma elevação discreta, estatisticamente significativa, da PMR para doenças do aparelho circulatório (PMR 114 ;IC 95\% 1,02-1,28). Foi também encontrado um discreto excesso de neoplasias entre os eletricitários, embora esse resultado não tenha alcançado significância estatística (PMR 1,11; IC 95\% 0,91-1,35). Para todas as demais causas de óbito, observou-se valores de PMR abaixo da unidade.

Tabela 1 - Distribuição das ocupações dos eletricitários segundo categorias de provável exposição aos campos magnéticos.

\begin{tabular}{lll}
\hline \multicolumn{1}{c}{ Alta exposição } & Média exposição & Baixa exposição \\
\hline Eletricista & Engenheiro & Supervisor \\
Feitor eletricista & Mecânico & Agente administrativo \\
Despachante de carga & Encarregado de oficina & Pintor \\
Operador de computadores & Caldereiro & Contador \\
& Assistente técnico & Chefe de seção \\
& Inspetor de carga & Escriturário \\
& Calibrador de medidores & Cozinheiro \\
& & Encadernador \\
& & Motorista \\
& & Ascensorista \\
& & Tapeceiro \\
\end{tabular}

Tabela 2 - Razão de Mortalidade Proporcional por causa (PMR) em eletricitários do Estado de São Paulo, 1975 - 1985.

\begin{tabular}{|c|c|c|c|c|}
\hline Causas de morte & Óbitos observados & Óbitos esperados & PMR & $95 \%$ \\
\hline Doenças infec. parasitárias & 11 & 17,87 & 0,61 & $0,30-1,09$ \\
\hline Neoplasmas & 105 & 94,18 & 1,11 & $0,91-1,35$ \\
\hline Doenças glând. endócrinas & 16 & 19,21 & 0,83 & $0,47-1,34$ \\
\hline Doenças sist. nerv. e órgãos dos sentidos & 3 & 5,14 & 0,58 & $0,11-1,69$ \\
\hline Doenças ap. circulatório & 287 & 252,28 & 1,14 & $1,02-1,28$ \\
\hline Doenças ap. respiratório & 28 & 52,00 & 0,54 & $0,36-0,78$ \\
\hline Doenças ap. digestivo & 35 & 37,84 & 0,92 & $0,64-1,28$ \\
\hline Doenças ap. gênito-urinário & 8 & 10,98 & 0,73 & $0,31-1,44$ \\
\hline Causas externas & 43 & 60,95 & 0,70 & $0,50-0,94$ \\
\hline Sint. e estados mórbidos maldef. & 28 & - & - & - \\
\hline
\end{tabular}

IC - Intervalo de Confiança 
Tabela 3 - "Cancer Mortality Odds Ratio" (CMOR) em eletricitários do Estado de São Paulo, 1975 - 1985.

\begin{tabular}{lccr}
\hline Faixa etária & CMOR & IC 95\% & \multicolumn{1}{c}{$p$} \\
\hline 20 a 29 anos & 1,86 & $\ldots$ & 0,31 \\
30 a 39 anos & 2,54 & $1,04-5,88$ & 0,02 \\
40 a 49 anos & 1,58 & $0,82-2,98$ & - \\
50 a 79 anos & 0,81 & $0,63-1,04$ & - \\
80 anos e mais & 1,07 & $0,41-2,63$ & 0,87 \\
Todas as idades & 0,95 & $0,76-1,17$ & - \\
\hline
\end{tabular}

IC - Intervalo de Confiança

Na Tabela 3 tem-se os resultados do "Mortality Odds Ratio" para câncer (CMOR). Não foi observado risco aumentado para câncer, considerando todas as idades em conjunto (CMOR 0,95; IC 95\% 0,761,17). Entretanto, para cada uma das faixas etárias consideradas, com exceção daquela que compreende os eletricitários de 50 a 79 anos de idade, observouse risco aumentado de câncer, embora não estatisticamente significativo. Para a faixa etária de 20-29 anos, não foi possível o cálculo do Intervalo de Confiança (IC), devido à presença de um valor muito pequeno em uma casela. Para as faixas etárias de 30-39 e 80 anos e mais, apresentou-se também o valor da probabilidade de ocorrência ao acaso do CMOR encontrado, uma vez que o IC foi considerado pouco preciso devido ao reduzido número de observações utilizadas para os cálculos.
Com relação aos diversos tipos específicos de câncer (Tabela 4), pôde-se observar que os neoplasmas de laringe apresentaram uma CPMR elevada e estatisticamente significativa. Outros tipos de câncer que mostraram CPMR elevadas, porém sem significação estatística, foram cavidade oral/faringe, próstata, bexiga, rim, cérebro e doença de Hodgkin. Para cavidade oral/faringe, bexiga e cérebro, o IC é assimétrico, estando desviado para a direita. Isso pode significar que, caso a amostra fosse maior, esse intervalo poderia não incluir a unidade.

Na Tabela 5, são descritos os resultados da análise da CPMR, segundo as categorias de provável exposição aos campos magnéticos.

No grupo de alta exposição, foram encontradas CPMRs elevadas e estatisticamente significativas, embora com um IC amplo, devido ao pequeno número de casos, para câncer de bexiga (CPMR 4,17; IC 95\% 1,35-9,72), cérebro (CPMR 3,77; IC 95\% 1,02-9,65) e doença de Hodgkin (CPMR 5,55; IC 95\% 1,14-16,21). Outros tipos específicos de câncer, para os quais foi observado um excesso de óbitos, porém não estatisticamente significativo, foram: cavidade oral/faringe, estômago, pâncreas e laringe. Com relação a câncer de cérebro, 4 dos 6 óbitos observados ocorreram no grupo com provável exposição elevada à eletricidade.

Tabela 4 - Razão de Mortalidade Proporcional por Câncer (CPMR), ajustada por idade, em eletricitários do Estado de São Paulo, 1975 - 1985.

\begin{tabular}{|c|c|c|c|c|}
\hline Tipo de câncer* & Óbitos observados & Óbitos esperados & PCMR & IC $\quad 95 \%$ \\
\hline Cav. oral/faringe (140-149) & 12 & 6,47 & 1,85 & $0,96-3,24$ \\
\hline Esôfago (150) & 7 & 8,01 & 0,87 & $0,35-1,79$ \\
\hline Estômago (151) & 18 & 22,98 & 0,78 & $0,64-1,23$ \\
\hline Intestino delgado (152) & 1 & 0,38 & 2,63 & $0,07-14,65$ \\
\hline Cólon (153) & 5 & 4,94 & 1,01 & $0,33-2,35$ \\
\hline Reto e ânus (154) & 1 & 2,73 & 0,37 & $0,01-2,06$ \\
\hline Fígado (155) & 2 & 3,22 & 0,62 & $0,07-2,24$ \\
\hline Pâncreas (157) & 4 & 4,63 & 0,86 & $0,23-2,20$ \\
\hline Laringe (161) & 12 & 5,89 & 2,04 & $1,05-4,20$ \\
\hline Pulmão (162) & 20 & 22,26 & 0,90 & $0,55-1,39$ \\
\hline Ossos (170) & 2 & 1,34 & 1,49 & $0,18-5,38$ \\
\hline Tecido conjuntivo (171) & 2 & 0,83 & 2,41 & $0,29-8,70$ \\
\hline Melanoma (172) & 1 & 1,22 & 0,82 & $0,02-4,57$ \\
\hline Próstata (185) & 10 & 8,28 & 1,21 & $0,58-2,23$ \\
\hline Testículo (186) & 1 & 0,37 & 2,70 & $0,07-15,04$ \\
\hline Bexiga (188) & 8 & 3,82 & 2,09 & $0,90-4,12$ \\
\hline $\operatorname{Rim}(189)$ & 4 & 1,50 & 2,67 & $0,73-6,83$ \\
\hline Cérebro (191-192) & 6 & 2,39 & 2,51 & $0,92-5,47$ \\
\hline Doença de Hodgkin (201) & 3 & 1,09 & 2,75 & $0,57-8,03$ \\
\hline Mieloma (203) & 1 & 0,94 & 1,06 & $0,03-5,90$ \\
\hline Não especificados & 10 & & & \\
\hline
\end{tabular}


Tabela 5 - Razão de Mortalidade Proporcional por Câncer (CPMR) segundo categorias de exposição aos campos magnéticos em eletricitários do Estado de São Paulo, 1975-1985.

\begin{tabular}{|c|c|c|c|c|c|c|c|c|c|}
\hline \multirow{2}{*}{ Tipo de Cancer* } & \multicolumn{3}{|c|}{ Alta exposição } & \multicolumn{3}{|c|}{ Média exposição } & \multicolumn{3}{|c|}{ Baixa exposição } \\
\hline & \multicolumn{3}{|c|}{ n CPMR (IC 95\%) } & \multicolumn{3}{|c|}{ n CPMR (IC 95\%) } & \multicolumn{3}{|c|}{ n CPMR (IC 95\%) } \\
\hline Cav. oral/faringe (140-149) & 5 & 1,81 & $(0,59-4,22)$ & 3 & 1,91 & $(0,39-5,58)$ & 4 & 1,61 & $(0,44-4,12)$ \\
\hline Esôfago (150) & 3 & 0,93 & $(0,19-2,72)$ & 1 & 0,48 & $(0,01-2,67)$ & 3 & 0,91 & $(0,19-2,66)$ \\
\hline Intestino delgado (152) & - & - & - & - & - & - & 1 & 8,33 & $(0,21-46,40)$ \\
\hline Cólon (153) & - & - & - & 2 & 1,60 & $(0,19-5,78)$ & 3 & 1,48 & $(0,30-4,32)$ \\
\hline Reto e ânus (154) & - & - & - & - & - & - & 1 & 0,55 & $(0,01-3,06)$ \\
\hline Fígado (155) & 1 & 0,83 & $(0,02-4,62)$ & - & - & - & 1 & 0,82 & $(0,02-4,57)$ \\
\hline Pâncreas (157) & 2 & 1,25 & $(0,15-4,51)$ & 1 & 0,90 & $(0,02-5,01)$ & 1 & 0,54 & $(0,01-3,01)$ \\
\hline Laringe (161) & 4 & 1,75 & $(0,48-4,48)$ & 2 & 1,38 & $(0,17-4,96)$ & 6 & 2,56 & $(0,94-5,58)$ \\
\hline Pulmão (162) & 4 & 0,51 & $(0,14-1,31)$ & 7 & 1,27 & $(0,51-2,62)$ & 8 & 0,90 & $(0,39-1,77)$ \\
\hline Ossos (170) & - & - & - & 2 & 7,69 & $(0,93-27,76)$ & - & - & - \\
\hline Tecido conjuntivo (171) & - & - & - & 1 & 7,69 & $(0,19-42,83)$ & 1 & 3,45 & $(0,09-19,22)$ \\
\hline Melanoma (172) & - & - & - & - & - & - & 1 & 2,00 & $(0,05-11,14)$ \\
\hline Próstata (185) & 3 & 1,22 & $(0,25-3,56)$ & - & - & - & 7 & 2,13 & $(0,85-4,39)$ \\
\hline Testículo (186) & - & - & - & - & - & - & 1 & 14,29 & $(0,36-79,59)$ \\
\hline Bexiga (188) & 5 & 4,17 & $(1,35-9,72)$ & 3 & 2,80 & $(0,58-8,18)$ & - & - & - \\
\hline $\operatorname{Rim}(189)$ & - & - & - & 1 & 2,86 & $(0,07-15,93)$ & 3 & 5,26 & $(1,08-15,36)$ \\
\hline Cérebro (191-192) & 4 & 3,77 & $(1,02-9,65)$ & 1 & 2,22 & $(0,06-12,36)$ & 1 & 1,10 & $(0,03-6,13)$ \\
\hline Doença de Hodgkin (201) & 3 & 5,55 & $(1,14-16,21)$ & - & - & - & - & - & - \\
\hline Mieloma (203) & 1 & 3,23 & $(0,08-17,99)$ & - & - & - & - & - & - \\
\hline Não especificados & 2 & - & - & 4 & - & - & 4 & - & - \\
\hline
\end{tabular}

IC - Intervalo de Confiança

* Número entre parênteses corresponde ao Código de Classificação Internacional de Doenças, 1975.

Tabela 6 - Razão de Mortalidade Proporcional por Câncer (CPMR) dos eletricitários de 20 a 79 anos, do Estado de São Paulo, ajustada por idade, em comparção com trabalhadores petroquímicos.

\begin{tabular}{|c|c|c|c|c|}
\hline Tipo de câncer* & Óbitos observados & Óbitos esperados & CPMR & $95 \%$ \\
\hline Cav. oral/faringe (140-149) & 11 & 9,15 & 1,20 & $0,60-2,15$ \\
\hline Esôfago (150) & 7 & 3,92 & 1,79 & $0,72-3,69$ \\
\hline Estômago (151) & 16 & 13,67 & 1,17 & $0,67-1,89$ \\
\hline Intestino delgado (152) & 1 & 0,64 & 1,56 & $0,04-8,69$ \\
\hline Cólon (153) & 5 & 2,35 & 2,13 & $0,69-4,96$ \\
\hline Reto e ânus (154) & 1 & 2,31 & 0,52 & $0,01-2,90$ \\
\hline Fígado (155) & 2 & 6,69 & 0,30 & $0,04-1,08$ \\
\hline Pâncreas (157) & 4 & 10,79 & 0,37 & $0,10-0,95$ \\
\hline Laringe (161) & 11 & 3,13 & 3,51 & $1,75-6,28$ \\
\hline Pulmão (162) & 18 & 20,85 & 0,86 & $0,51-1,36$ \\
\hline Ossos (170) & 2 & 0,78 & 2,56 & $0,31-9,24$ \\
\hline Tec. conjuntivo (171) & 2 & 0,30 & 6,67 & $0,8-24,08$ \\
\hline Melanoma (172) & 1 & - & - & $-\quad-$ \\
\hline Próstata (185) & 10 & 6,67 & 1,50 & $0,72-2,76$ \\
\hline Testículo (186) & 1 & - & - & $-\quad-$ \\
\hline Bexiga (188) & 7 & 0,93 & 7,53 & $3,02-15,51$ \\
\hline $\operatorname{Rim}(189)$ & 4 & 2,99 & 1,34 & $0,36-3,43$ \\
\hline Cérebro (191-192) & 6 & 8,15 & 0,74 & $0,27-1,61$ \\
\hline D. Hodgkin (201) & 3 & 1,77 & 1,69 & $0,34-4,93$ \\
\hline Mieloma (203) & 1 & 1,71 & 0,58 & $0,01-3,23$ \\
\hline Não especificados & 10 & - & - & $-\quad-$ \\
\hline
\end{tabular}

IC - Intervalo de Confiança

* Número entre parênteses corresponde ao Código Internacional de Doenças, 1975. 
Para os eletricitários com provável exposição moderada aos campos eletromagnéticos foi encontrado excesso de óbitos por vários tipos de câncer, embora nenhum deles tenha sido estatisticamente significativo.

No grupo de baixa exposição, foi encontrada uma elevação da CPMR para neoplasmas de rim, estatisticamente significativa, embora também com um IC amplo (CPMR 5,26; IC 95\% 1,08-15,36). Outros CPMRs elevados, porém sem significância estatística, foram cavidade oral/faringe, cólon, laringe e próstata. Uma vez que todos os indivíduos incluídos no grupo de baixa exposição a campos magnéticos são trabalhadores administrativos, outros fatores de risco devem ser considerados para a análise da distribuição de câncer entre os mesmos.

Na Tabela 6 são apresentados os resultados da análise da CPMR utilizando, como comparação, trabalhadores petroquímicos, sendo encontrada uma elevação significativa de óbitos por câncer de laringe (CPMR 3,51; IC 1,75-6,28) e bexiga (CPMR 7,53; IC 95\% 3,02-15,51). Outras localizações anatômicas com CPMRs elevados, porém estatisticamente não significativos, foram cavidade oral/faringe, esôfago, estômago, cólon, ossos, tecido conjuntivo, próstata, rim e doença de Hodgkin.

\section{DISCUSSÃO}

A utilização da categoria ocupacional, como medida indireta de exposição aos CEM tem sido bastante criticada na literatura ${ }^{12,31,32,61}$. No presente trabalho, a não disponibilidade de dados quantitativos de exposição aos campos magnéticos, para o conjunto de trabalhadores estudados, levou a utilizar a ocupação como medida indireta indicativa, ou não, da exposição de cada indivíduo.

A categoria "eletricitário" constitui, sem dúvida, um grupo bastante heterogêneo, consistindo em um conjunto de indivíduos expostos a diferentes intensidades de CEM e, outros, inclusive, sem exposição marcadamente diferenciada daquela existente na população em geral (Tabela 1). Embora uma medida de exposição mais precisa seja sempre desejável, o conjunto de informações atualmente disponível permite avaliar, com um pouco mais de segurança, os resultados obtidos em estudos que utilizam medidas indiretas, como é o caso da categoria ocupacional ${ }^{39}$, ${ }^{40}$. Por outro lado, os efeitos desse tipo de classificação da exposição, de modo geral, costumam levar a uma subestimação do risco real ${ }^{13,22}$.

Peculiaridades inerentes aos equipamentos utilizados na geração, transmissão e distribuição de energia elétrica em cada localidade, associadas às diferentes características da população trabalhadora, tornam importante verificar, no Brasil, a possibilidade de ocorrência da associação entre exposição aos CEM no setor elétrico e o desenvolvimento de câncer. Os únicos dados disponíveis para o Brasil $^{39}$ mostraram resultados relativamente semelhantes aos encontrados nos diversos estudos epidemiológicos já publicados.

A metodologia utilizada para esse estudo merece alguns comentários. A Razão Padronizada de Mortalidade (SMR) é a base de inferência preferida em estudos de mortalidade ocupacional e, em contraste, a PMR é considerada, de modo geral, como uma metodologia mais deficiente ${ }^{52,84}$. A não-comparabilidade entre coortes ocupacionais e populações de referência é amplamente reconhecida, refletindo-se no Efeito do Trabalhador Sadio ("Health Worker Effect" HWE) ${ }^{16,83}$. Devido à influência do HWE, os estudos epidemiológicos de grupos ocupacionais, frequientemente encontram taxas de mortalidade mais baixas do que aquelas da população em geral e podem, assim, deixar de detectar doenças relacionadas à ocupação ${ }^{84}$. Na forma mais simples de HWE, os trabalhadores industriais costumam ter uma mortalidade menor do que a esperada por doenças cardiovasculares e do aparelho respiratório ${ }^{55}$. Estudos de coortes de trabalhadores mostram que, no período inicial de seguimento, os menores riscos relativos encontrados são para as doenças respiratórias e do aparelho digestivo ${ }^{53}$. Outros autores mencionam também as doenças das glândulas endócrinas e dos aparelhos digestivo e gênito-urinário ${ }^{10}$.

No grupo estudado constatou-se que as PMRs relativas a doenças respiratórias e dos aparelhos digestivo e gênito-urinário apresentaram valores abaixo de $100 \%$, mas o mesmo não aconteceu com as doenças cardiovasculares (Tabela 2). Esse resultado é bastante curioso, uma vez que representa um comportamento contrário ao previsto na literatura. É importante lembrar, porém, que esse grupo de causas de morte contribuiu com a metade dos óbitos da amostra, o que poderia ter influenciado o resultado encontrado $^{84}$. São poucos os estudos sobre efeitos da exposição aos CEM relacionados a outra patologia que não o câncer. Referências a alterações fisiológicas do aparelho circulatório são encontradas em trabalhos russos da década de $60^{6}$, não tendo sido, porém, confirmados em estudos posteriores ${ }^{37,66}$.

Com relação a câncer, os resultados são relativamente consistentes com os do único estudo brasilei$\mathrm{ro}^{39}$, assim como com os de diversos outros trabalhos publicados $^{34,44,50,56,75}$. É necessário salientar, entre- 
tanto, importantes diferenças metodológicas entre este trabalho e os estudos citados. A heterogeneidade da amostra, formada por indivíduos expostos a intensidades diferentes de campos magnéticos, por períodos diversos de tempo e, por indivíduos não expostos, precisa ser lembrada como um fator que poderia levar à subestimação dos valores observados. Esse fato deve também ser considerado em relação aos resultados do CMOR. A hipótese a priori desse estudo, de acordo com a literatura, não era a de encontrar uma associação entre exposição aos CEM de freqüência industrial e todos os tipos de câncer e, sim, com tumores de localizações específicas, particularmente leucemias e tumores de cerébro, citados com maior freqüência na literatura $^{44,56,59,61,62,63,72}$.

Para evitar o viés que é introduzido em um estudo ocupacional, sempre que o grupo de comparação é a população em geral, foi proposta a utilização da Razão Padronizada de Mortalidade Específica (SPMR), como uma metodologia menos sujeita a esse tipo de erro ${ }^{84}$. As doenças neoplásicas, em especial, costumam ser menos afetadas pelo HWE, pois parece não existir uma forma de seleção que possa identificar, a priori, os indivíduos que virão a desenvolver essa patologia ${ }^{20}$. Assim, a comparação entre a proporção de óbitos por tipos específicos de câncer, em determinado grupo de trabalhadores, e a proporção por essa mesma doença encontrada em uma população de referência, tende a apresentar resultados mais fidedignos do que aqueles observados para a PMR. Diversos autores assinalam que o HWE praticamente não interfere em estudos de mortalidade por câncer, dando como justificativa o fato de que as neoplasias constituem um conjunto de doenças que ocorrem em faixas etárias mais velhas, ou cujos sintomas surgem pouco antes da morte, ou para as quais não existem métodos diagnósticos capazes de prever seu futuro aparecimento, no momento da seleção para o emprego ${ }^{9,10,79}$. Uma limitação que precisa ser destacada, porém, é o número de óbitos de câncer estudados, insuficiente para proporcionar estabilidade aos resultados estatísticos; esse fato evidencia-se nos intervalos de confiança muito amplos das CPMR e na falta de significância estatística de quase todos os resultados.

A impossibilidade de obter informações sobre outros fatores de risco, inclusive aqueles já estabelecidos, como fumo e álcool, precisa ser reconhecida como uma importante limitação para a interpretação desses resultados. Alguns tipos específicos de câncer, para os quais foi observado um excesso de óbitos entre os eletricitários, como cavidade oral/faringe, laringe e bexiga, têm o fumo como importante fator de risco ${ }^{11}$. Os resultados para câncer de cérebro, contudo, não poderiam ser explicados facilmente pela presença desse tipo de fator de risco. Esse é também o caso da doença de Hodgkin, embora o número de óbitos envolvidos seja muito pequeno para permitir qualquer tipo de conclusão.

Numa tentativa de diminuir a heterogeneidade do grupo, permitindo uma análise mais detalhada, os indivíduos do estudo foram classificados em categorias de exposicão estimada aos campos magnéticos, tendo como referência dados de medidas de intensidade de exposição provenientes de um trabalho realizado em diversos países do mundo ${ }^{39}$. Esse procedimento pode ter introduzido um viés de classificação, pois a intensidade de exposição, para cada tipo de ocupação, pode variar de um país para o outro, não sendo necessariamente a mesma que ocorria em São Paulo. Entretanto, como a proposta do presente trabalho era uma análise exploratória dos dados, julgou-se que os resultados dessa classificação tenham sido satisfatórios.

Outra consideração importante é o fato de que só foi possível analisar a última ocupação do indivíduo, o que não corresponde, necessariamente, à ocupação exercida ao longo de todo o período de trabalho. $O$ viés de classificação não diferencial, contudo, costuma levar, conforme já mencionado, à subestimação do risco, e não à superestimação.

As CPMRs obtidas para os diferentes grupos de exposição mostraram-se consistentes com os resultados obtidos para o conjunto de eletricitários. Particularmente interessante é a concentração de quase todos os óbitos de câncer de cérebro na categoria de alta exposição, assim como a distribuição das mortes por câncer de bexiga apenas nos grupos de alta e média exposição. Os óbitos por doença de Hodgkin, embora em pequeno número, se concentraram também na categoria de maior exposição (Tabela 5). Por outro lado, como já foi mencionado, diversos trabalhos têm mostrado uma associação entre fumo ou álcool e câncer de bexiga ${ }^{10,11,49}$, e esses antecedentes não são conhecidos para o grupo de eletricitários, o que não permite afastá-los como possíveis fatores de distorção dos resultados.

No seu ambiente de trabalho, as diferentes categorias ocupacionais expostas aos CEM de frequiência industrial entram em contato com inúmeras substâncias químicas (vapores metálicos, bifenilas policlorinadas, solventes orgânicos), as quais têm sido sugeridas como possíveis fatores de distorção da associação em estudo ${ }^{34,56,62,71,72}$. No roteiro de exames periódicos de eletricitários existem algumas indicações sobre as substâncias químicas a que os 
mesmos se encontram expostos no desempenho de suas atividades. As categorias de alta e média exposição aos CEM, na classificação utilizada, envolvem grupos de indivíduos expostos também à anilina e ao nitrobenzeno, substâncias mencionadas em vários estudos epidemiológicos como associadas ao câncer de bexiga ${ }^{27,41}$. Alguns estudos epidemiológicos mostraram evidências de uma relação entre câncer de cérebro e exposição a solventes orgânicos ${ }^{59,72}$. Com relação aos compostos clorados, produtos utilizados em transformadores, um estudo epidemiológico encontrou excesso significativo de câncer de cérebro em indivíduos expostos ${ }^{67}$.

Entre os indivíduos considerados como pertencentes ao grupo de baixa exposição, a grande maioria são funcionários administrativos, e outros fatores de risco devem ser considerados para a análise da distribuição de câncer entre os mesmos.

A comparação da experiência de mortalidade por neoplasias dos eletricitários de São Paulo, com uma população de trabalhadores petroquímicos (Tabela 6), embora diminua a influência do HWE, não elimina totalmente o efeito de fatores como nível educacional, ou socioeconômico, entre ou$\operatorname{tros}^{79}$, devido a diferenças nos respectivos processos de seleção; essas diferenças precisam ser lembradas como possíveis fatores explicativos dos resultados encontrados.

Observou-se, nessa análise, que as PCMRs, com exceção do câncer de cérebro, mantêm-se elevadas para as mesmas localizações anatômicas encontradas, quando a população de referência era a do Município de São Paulo, o que parece reforçar a possibilidade de uma associação entre sua ocorrência e a exposição, em maiores níveis, aos CEM. Entretanto, um excesso de óbitos por câncer de cérebro foi encontrado entre a população de trabalhadores petroquímicos utilizada como referência para essa análise ${ }^{47}$. Alguns trabalhos têm mostrado evidências de que a associação entre trabalho na indústria petroquímica e câncer de cérebro se dê através da exposição ao benzeno e outros solventes orgânicos, também presentes no ambiente de trabalho dos eletricitários $^{59,73}$.

Embora não tenha sido ainda demonstrado, de maneira inequívoca, o mecanismo de ação através do qual os CEM exerceriam seu papel no processo de carcinogênese, estudos experimentais têm evidenciado que eles não exercem um efeito direto sobre o DNA, ao contrário do que acontece com diversos agentes iniciadores; parece, assim, mais provável que seu efeito ocorra de outra maneira, por exemplo, na fase de promoção tumoral ${ }^{19,69,70,78}$. A possibilidade de uma ação promotora dos CEM de 50-60 Hz implica considerar a importância da presença de outros fatores carcinógenos potenciais no ambiente de trabalho dos eletricitários, uma vez que um agente promotor atua de forma seqüencial, após a exposição a um iniciador ${ }^{7}$.

Evidências de estudos epidemiológicos favorecem essa possibilidade. Em seu estudo de exposição residencial a fontes de alta tensão e câncer em adultos, Wertheimer e Leeper ${ }^{81}$ encontraram resultados positivos, utilizando o endereço mais recente dos indivíduos, o que indicaria um efeito dos CEM em etapas mais tardias da carcinogênese. Lin e col. ${ }^{44}$ analisaram diversos estudos ocupacionais que haviam encontrado um excesso de tumores de cérebro em trabalhadores com possível exposição à eletricidade; na maioria desses estudos, os casos de tumor de cérebro mostraram, em relação ao início da exposição, um período de latência curto e apareceram, de forma mais freqüente, em trabalhadores jovens. Segundo os autores, esses resultados são indicativos de uma ação promotora dos CEM, pois a exposição aos mesmos só seria efetiva já no final do processo de carcinogênese.

A duração do período de latência varia para os diversos tipos de câncer ${ }^{60}$. Assim, certo tipo de neoplasia pode aparecer em épocas mais tardias da vida por que, para que ocorra a iniciação, é necessária uma longa exposição a um determinado fator de risco; outras, entretanto, podem ocorrer mais precocemente, caso um período curto de exposição seja suficiente para o início do processo de carcinogênese. $\mathrm{Ou}$, ainda, o fator de risco que desencadeia a etapa de iniciação pode ser um carcinógeno ambiental bastante comum e, por isso, o contato com o mesmo ocorreria em idades mais jovens ${ }^{9,75}$.

Se as colocações acima indicadas forem verdadeiras, os diversos fatores de risco a que os eletricitários se encontram expostos, no seu ambiente de trabalho, podem atuar, de forma seqüencial, no desenvolvimento de neoplasias. Dependendo do tipo de câncer envolvido, essa seqüência poderia ser diferente e envolver agentes diversos, culminando no aparecimento do tumor, após um período de latência curto, ou longo. Os CEM parecem atuar em etapas mais tardias do processo de carcinogênese e, nesse caso, exposições que ocorressem fora desse estágio particular do processo de desenvolvimento da doença, seriam de pouca relevância, pois não teriam ação sobre o mesmo. Entretanto, caso esses indivíduos fossem incluídos entre os expostos, contribuiriam para uma diluição da associação. Essa poderia ser uma possível explicação para as discrepâncias observadas, com 
relação ao tipo de câncer envolvido, entre os estudos epidemiológicos de exposição ocupacional, até então publicados.

O estudo dos efeitos da exposição aos CEM em várias "janelas" de tempo, ou seja, em períodos de exposição de diferentes durações, associado à análise dos diferentes fatores potenciais de risco presentes no ambiente de trabalho, contribuiria, certamente, para desvendar novos aspectos do papel dos mesmos no processo de carcinogênese.

\section{REFERÊNCIAS BIBLIOGRÁFICAS*}

1. AHLBOM, A. A review of the epidemiologic literature on magnetic fields and cancer. Scand. J. Work Environ. Health, 14:337-43, 1988.

2. ARMANINI, D.; CONTI, R.; MANTINI, A.; NICOLINI, P.; DI LECCE, F.; LATTARULO, F. Mastronardi G. Enel's experience in the area of power-frequency electromagnetic field measuring techniques: problems related to design, calibration, and use of measuring devices. Alta Frequenza, 58:403-10, 1989.

3. BASTUJI-GARIN, S.; RICHARDSON, S.; ZITTOUN, R. Acute leukaemia in workers exposed to electromagnetic fields. Eur. J. Cancer, 26:1119-20, 1990.

4. BATES, M. N. Extremely low frequency electromagnetic fields and cancer: the epidemiologic evidence. Environ. Health Perspect., 95:147-56, 1991.

5. BISHOP, J. M. The molecular genetics of cancer. Leukemia, 2:199-208, 1988.

6. BONNELL, J. A. Effects of electric fields near powertransmission plant. J. R. Soc. Med., 75:933-41, 1982.

7. BRANDT-RAUF, P. W. Oncogene proteins as biomarkers in the molecular epidemiology of occupational carcinogenesis. Int. Arch. Occup. Environ. Health, 63:1-8, 1991.

8. CALLE, E. E. \& SAVITZ, D. A. Leukemia in occupational groups with presumed exposure to electrical and magnetic fields. N. Engl. J. Med., 313:1476-7, 1985.

9. CHECKOWAY, H.; PEARCE, N.; CRAWFORD-BROWN, D. J. Research methods in occupational epidemiology. New York, Oxford University Press, 1989.

10. CHOI, B. C. K. Definition, sources, magnitude, effect modifiers, and strategies of reduction of the healthy worker effect. J. Occup. Med., 34:979-88, 1992.

11. CLAUDE, J.; KUNZE, E.; FRENTZEL-BEYME, R.; PACZKOWSKI, K.; SCHNEIDER, J.; SCHUBERT, H. Lifestyle and occupational risk factors in cancer of the lower urinary tract. Am. J. Epidemiol., 124: 578-89, 1986.
Em que pesem as restrições mencionadas sobre o tamanho do grupo de estudo e a ausência de informações sobre possíveis fatores de distorção, os resultados do presente estudo mostraram-se relativamente similares aos descritos na literatura. Nesse sentido, representam mais um conjunto de resultados que reforçam a possibilidade de uma associação causal entre exposição aos CEM de frequiência industrial e o desenvolvimento de alguns tipos específicos de câncer.

12. COLEMAN, M. \& BERAL, V. A review of epidemiological studies of the health effects of living near or working with electricity generation and transmission equipment. Int. J. Epidemiol., 17:1-13, 1988.

13. COPELAND, K. T.; CHECKOWAY, H.; MCMICHAEL, A. J.; HOLBROOK, R. H. Bias due to misclassification in the estimation of relative risk. Am. J. Epidemiol., 105:488-95, 1977.

14. DAN BRACKEN, T. Exposure assessment for power frequency electric and magnetic fields. Presented in Panel: Electric and Magnetic Fields and Health. Paris, 1992.*

15. DEADMAN, J. E.; SCHNITMAN, A.; THÉRIAULT, G. Reconstruction of past exposures to electric and magnetic fields. In : Joint Électricité de France, Hydro-Québec, Ontario Hydro Epidemiological Study on the Longterm Effects of Exposure to 50 and 60 Hertz Electric and Magnetic Fields, 1991.*

16. DECOUFLÉ, P.; THOMAS, T. L.; PIKLE, L. Comparison of the proportionate mortality ratio and standardized mortality ratio risk measures. Am. J. Epidemiol., 111:263-9, 1980.

17. DELPIZZO, V.; SALZBERG, M. R.; FARISH, S. J. The use of "spot" measurements in epidemiological studies of the health effects of magnetic field exposure. Int. J. Epidemiol., 20: 448-55, 1991.

18. DUBROW. R. \& WEGMAN, D. H. Cancer and occupation in Massachusetts: a death certificate study. Am. J. Ind. Med., 6:207-30, 1984.

19. EASTERLY, C. E. Cancer link to magnetic field exposure: a hypothesis. Am. J. Epidemiol., 114:169-74, 1981.

20. ENTERLINE, F. E. Not uniformly true for each cause of death. J. Occup. Med., 17:127-8, 1975.

21. FEYCHTING, M. \& AHLBOM, A. Magnetic fields and cancer in people residing near Swedish high voltage power lines. Am. J. Epidemiol., 138:467-81, 1993.

22. FLEGAL, K. M.; BROWNIE, C.; HAAS, J. D. The effects of exposure misclassification on estimates of relative risks. Am. J. Epidemiol., 123:736-51, 1986. 
23. FLODERUS, B. \& KNAVE B. Occupational exposure to electromagnetic fields in relation to leukemia and brain tumors: a case-control study in Sweden. Cancer Causes Control., 4:465-76, 1993.

24. FLODIN, U.; FREDRIKSSON, M.; AXELSON, A.; PERSSON, B.; HARDELL, L. Background radiation, electrical work, and some other exposures associated with acute myeloid leukemia in a case-referent study. Arch. Environ. Health, 41:77-84, 1986.

25. FUNDAÇÃO SEADE. Causas de morte e esperança de vida ao nascer no Estado de São Paulo e regiões - 1975-1983. São Paulo, 1990.

26. GARLAND, F. C.; SHAW, E.; GORHAM, E. D.; GARLAND, C. F.; WHITE, M. R.; SINSHEIMER, P. J. Incidence of leukemia in occupations with potential electromagnetic field exposure in Unites States Navy personnel. Am. J. Epidemiol., 132:293-303, 1990.

27. GONZALEZ, C. A.; LÓPEZ-HABENTE, G.; ERREZOLA, M.; ESCOLAR, A.; RIBOLI, E.; IZARZUGAZA, I.; NEBOT, M. Occupation and bladder cancer in Spain: A multi-centre case-control study. Int. J. Epidemiol., 18:56977, 1989.

28. GOODMAN, R.; BASSETT, CAL.; HENDERSON, A. S. Pulsing electromagnetic fields induce cellular transcription. Science, 220:1283-5, 1983.

29. HERMO JR, H. Chemical carcinogenesis: tumor initiation and promotion. In: Emmelt, E. A., ed. Health problems of health care workers. Philadelphia, Harley \& Belfus, 1987. p. 1-25 (Ocupational medicine: state of the art reviews, 2).

30. INTERNATIONAL ELECTRICITY RESEARCH EXCHANGE (IERE). Epidemiological studies relating human health to electric and magnetic fields: criteria for evaluation. 1988. [Report]*

31. JAUCHEM, J. R. \& MERRIT, J. H. The epidemiology of exposure to electromagnetic fields: an overview of the recent literature. J. Clin. Epidemiol., 44:895-906, 1991.

32. JAUCHEM, J. R. Epidemiologic studies of electric and magnetic fields and cancer: a case study of distortions by the media. J. Clin. Epidemiol., 45:1137-42, 1992.

33. JUNQUEIRA, L. A. P.; EDUARDO, M. B. P.; SOBOLL, M. L. M.; INOJOSA, R. M.; JARDANOVSKI, E.; ARAÚJO, L. C. M. N. Gestão pública no setor saúde: um plano estratégico. São Paulo, FUNDAP, 1992.

34. JUUTILAINEN, J.; LAARA, E.; PUKKALA, E. Incidence of leukaemia and brain tumours in Finnish workers exposed to ELF magnetic fields. Int. Arch. Occup. Environ. Health, 62:289-93, 1990.

35. KAUNE, W. T.; STEVENS, R. G.; CALLAHAN, N. J.; SEVERSON, R. K.; THOMAS, D. B. Residential magnetic and electric fields. Bioelectromagnetics, 8:315-35, 1987.

36. KAVET, R.; SILVA, J. M.; THORNTON, D. Magnetic field exposure assessment for adult residents of Maine who live near and far away from overhead transmission lines. Bioelectromagnetics, 13:35-55, 1992.
37. KNAVE, B.; GAMBERALE, F.; BERGSTROM, S.; BIRKE, E.; IREGREN, A.; KOLMODIN-HEDMAN, B.; WENNBERG, A. Long-term exposure to electric fields - a cross-sectional epidemiologic investigation of occupationally exposed workers in high-voltage substations. Scand. J. Environ. Health, 5:115-25, 1979.

38. KOIFMAN, S. Electromagnetic fields: a cancer promoter? Med. Hypothesis, 41:23-7, 1993.

39. KOIFMAN, S.; ECHENIQUE, I.; FARIAS, A.; MONTEIRO, G.; KOIFMAN, R. Cancer mortality among workers in an electrical company in Rio de Janeiro, Brazil. In: Procedings of the Symposium on Environmental Stressors, Tampere, 1989*.

40. KOIFMAN, S. \& THERIAULT, G. Electric and magnetic fields and cancer: The use of field exposure measurements in epidemiological studies. In: Carpenter, D. O. \& Ayrapetyan, S., ed. Biological effects of electric and magnetic fields. San Diego, Academic Press, 1994. v. 2, p. 201-31.

41. LA VECCHIA, C.; NIGRI, E.; D’ AVANZO, B.; FRANCESCHI, S. Occupation and the risk of bladder cancer. Int. J. Epidemiol., 119:264-8, 1990.

42. LIBOFF, A. R.; WILLIAMS, T.; STRONG, D. M.; WISTAR, R. Time-varying magnetic fields: effect on DNA synthesis. Science, 223:818-20, 1984.

43. LILIENFELD, A. M. \& LILIENFELD, D. E. Fundamentos de epidemiologia. Bogotá, Fondo Educativo Interamericano, 1983.

44. LIN, R. S.; DISCHINGER, P. C.; CONDE, J.; FARREL, K. P. Occupational exposure to electromagnetic fields and the occurrence of brain tumors. J. Occup. Med., 27:413-9, 1985.

45. LONDON, S. J.; THOMAS, D. C.; BOWMAN, J. D.; SOBEL, E.; CHENG, T. C.; PETERS, J. M. Exposure to residential electric and magnetic fields and risk of childhood leukemia. Am. J. Epidemiol., 134:923-37, 1991.

46. MADDOCK, B. J. Guidelines and standards for exposure to electric and magnetic fields at power frequencies. Panel: Electric and Magnetic Fields and Health. CIGRE Session Papers, 1992.*

47. MARTINS, J. F. P. Mortalidade por câncer em trabalhadores da indústria do petróleo - Brasil - 1970 a 1985. Rio de Janeiro, 1991. [Dissertaçao de Mestrado - Escola Nacional de Saúde Pública].

48. MATANOSKI, G. M.; BREYSSE, P. N.; ELLIOTT, E. A. Electromagnetic field exposure and male breast cancer. Lancet, 337:737, 1991.

49. MIETTINEN, O. S. \& DER WANG, J. An alternative to the proportionate mortality ratio. Am. J. Epidemiol., 114:144-8, 1981.

50. MILHAM JR., S. Mortality from leukemia in workers exposed to electrical and magnetic fields. N. Engl. J. Med., 307:249, 1982.

51. MINISTÉRIO DA SAÚDE. Estatísticas de mortalidade Brasil. Brasília, 1977 - 1985.

52. MONSON, R. R. Occupational epidemiology. Boca Raton, CRC Press, 1980. 
53. MONSON, R. R. Observations on the healthy worker effect. J. Occup. Med., 28:425-37, 1986.

54. OLIN, R.; VAGERO, D.; AHLBOM, A. Mortality experience of electrical engineers. Br. J. Ind. Med., 42:211-2, 1985.

55. PARK, R. M.; MAIZLISH, N. A.; PUNNETT, L.; MOUREERASO, R.; SILVERSTEIN, M. A. A comparison of PMRs and SMRs as estimators of occupational mortality. (Epidemiology), 2:49-59, 1991.

56. PEARCE, N.; REIF, J.; FRASER, J. Case-control studies of cancer in New Zealand electrical workers. Int. J. Epidemiol., 18:55-9, 1989.

57. PIROTTE, P. Some facts about E and B fields at the power frequencies. Panel: Electric and Magnetic Fields and Health. CIGRE Session Papers, 1992.*

58. POOLE, C. \& TRICHOPOULOS, D. Extremely lowfrequency electric and magnetic fields and cancer. Cancer Causes and Control, 2:267-76, 1991.

59. REIF, J. S.; PEARCE, N.; FRASER, J. Occupational risks for brain cancer: a New Zealand cancer registry-based study. J. Occup. Med., 31:863-7, 1989.

60. ROTHMAN, K. J. Induction and latent periods. Am. J. Epidemiol., 114:253-9, 1981.

61. SAVITZ, D. A. \& CALLE, E. E. Leukemia and occupational exposure to electromagnetic fields: review of epidemiologic surveys. J. Occup. Med., 29:47-51, 1987.

62. SAVITZ, D. A.; PEARCE, N. E.; POOLE, C. Methodological issues in the epidemiology of electromagnetic fields and cancer. Epidemiol. Rev., 11:59-78, 1989.

63. SAVITZ, D. A.; PEARCE, N.; POOLE, C. Update on methodological issues in the epidemiology of electromagnetic fields and cancer. Epidemiol. Rev., 15:558-66, 1993.

64. SAVITZ, D. A.; WATCHEL, H.; BARNES, F. A.; JOHN, E. M.; TVRDIK, J. G. Case-control study of childhood cancer and exposure to $60 \mathrm{~Hz}$ magnetic fields. Am. J. Epidemiol., 128:21-38, 1988.

65. SHEIKH, K. Exposure to electromagnetic fields and the risk of leukemia. Arch. Environ. Health, 41:56-63, 1986.

66. SINGEWALD, M. L.; LANGWORTHY, O. R.; KOUWENHOVEN, W. B. Medical follow-up of high voltage linemen working in AC electric fields. Presented in: IEEE PES Winter Meeting, New York, 1973.*

67. SINKS, T.; STEELE, G.; SMITH, A. B.; WATKINS, K.; SHULTS, R. A. Mortality among workers exposed to polychlorinated biphenyls. Am. J. Epidemiol., 136:389-98, 1992.

68. STERN, F. B.; WAXWEILER, R. A.; BEAUMONT, J. J.; LEE, S. T.; RINSKY, R. A.; ZUMWALDE, R. D.; HALPERIN, W. E.; BIERBAUM, P. J.; LANDRIGAN, P. J.; MURRAY, J. R. W. E. A case-control study of leukemia at a naval nuclear shipyard. Am. J. Epidemiol., 123:980-92, 1986.

69. STEVENS, R. Electric power use and breast cancer: a hypothesis. Am. J. Epidemiol., 125:556-61, 1987.
70. TENFORDE, T. S. Biological interactions and potential health effects of extremely-low-frequency magnetic fields from power lines and other common sources. Ann. Rev. Public Health, 13:173-96, 1992.

71. THÉRIAULT, G. Champs électromagnétiques et risques de cancer. Arch. Mal. Prof., 53:535-40, 1992.

72. THOMAS, T. L.; STOLLEY, P. D.; STEMHAGEN, A.; FONTHAM, E. T. H.; BLEEKER, M.; STEWART, P. A HOOVER, R. N. Brain tumor mortality risk among men with electrical and electronics jobs: a case-control study. $J$. Natl. Cancer Inst., 79:233-8, 1987.

73. THOMAS, T. L. \& WAXWEILER, R. J. Brain tumors and occupational risk factors. Scand. J. Work Environ. Health, 12:1-15, 1986.

74. TORNQVIST, S.; NORELL, S.; AHLBOM, A.; KNAVE, B Cancer in the electric power industry. Br. J. Ind. Med., 43:221-3, 1986

75. TYNES, T.; ANDERSEN, A.; LANGMARK, F. Incidence of cancer in Norwegian workers potentially exposed to electromagnetics fields. Am. J. Epidemiol., 136:81-8, 1992.

76. VAGERO, D. \& OLIN, R. Incidence of cancer in the electronics industry: using the new Swedish Cancer Environment Registry as a screening instrument. Br. J. Ind. Med., 40:188-92, 1983.

77. VAGERO, D.; AHLBOM, A.; OLIN, R.; SAHLSTEN, S. Cancer morbidity among workers in the telecommunications industry. Br. J. Ind. Med., 42:191-5,1985.

78. WALBORG JR., E. F. Extremely low frequency electromagnetic fields and cancer: focus on tumor initiation, promotion, and progression. Dermigen, 1991.*

79. WEED, D. L.; TYROLER, H. A.; SHY, C. The healthy worker effect in actively working communications workers. J. Occup. Med., 29:335-9, 1987.

80. WERTHEIMER, N. \& LEEPER, E. Electrical wiring configurations and childhood cancer. Am. J. Epidemiol., 109:273-84, 1979.

81. WERTHEIMER, N. \& LEEPER, E. Adult cancer related to electrical wirings near the home. Int. J. Epidemiol., 11:34555,1982 .

82. WIKLUND, K.; EINHORN, J.; EKLUND, G. An application of the Swedish Cancer Environment registry: Leukaemia among telephone operators at the telecommunications administration in Sweden. Int. J. Epidemiol., 10:373-6, 1981.

83. WILCOSKY, T. \& WING, S. The healthy worker effect selection of workers and work forces. Scand. J. Environ. Health, 13:70-2 , 1987.

84. WONG, O. \& DECOUFLÉ, P. Methodological issues involving the standardized mortality ratio and proportionate mortality ratio in occupational studies. J. Occup. Med., 24:299-304, 1982.

85. WRIGHT, W. E.; PETERS, J. M.; MACK, T. M. Leukaemia in workers exposed to electrical and magnetic fields. [Letter] Lancet, 2: (8308):1160-1, 1982. 